\title{
Planejamento familiar: uso dos serviços de saúde por jovens com experiência de gravidez
}

\author{
Family planning: use of the health services \\ by young people with experience of pregnancy
}

Laís Norberta Bezerra de Moura ${ }^{1}$

Keila Rejane Oliveira Gomes ${ }^{1}$

${ }^{1}$ Programa de Pós-

Graduação em Ciências e Saúde, Universidade Federal do Piauí. Av. Frei Serafim 2280/Centro de Ciências da Saúde. 64.000-020 Teresina PI Brasil.

lais_nurse@hotmail.com
Abstract The scope of this paper was to analyze the use of family planning services by young women with experience of pregnancy. It involves a cross-sectional study conducted with 464 young women who completed a pregnancy in the first quarter of 2006 in six hospitals in Teresina, when they were between 15 and 19 years of age. Data were collected in 2008 through a pre-coded and pre-tested form. Multivariate analysis showed that young women with lower income and a higher number of pregnancies, for whom health professionals, family and partner were their source of information, and who used contraception in their last sexual relations, were more likely to be users of family planning services. Approximately $55 \%$ of young women said they would seek a specialized service for their age group, even if it was far from their residence. It was revealed that the$r e$ is a need for investments in a specialized service with inherent characteristics that facilitate early access for young women, and also address the concerns of the young women who, despite having experienced an adolescent pregnancy, do not use the available family planning services.

Key words Health services, Family planning, Adolescent pregnancy
Resumo Objetivou-se analisar o uso de serviços de planejamento familiar por jovens com experiência de gravidez. Trata-se de estudo transversal realizado com 464 jovens que finalizaram uma gravidez no primeiro quadrimestre de 2006, quando tinham entre 15 e 19 anos, em seis maternidades de Teresina. Os dados foram coletados em 2008, por meio de formulário pré-codificado e pré-testado. A análise multivariada apontou que jovens de menor renda, maior número de gestações, que têm como fonte de informação profissionais de saúde, família e parceiro, e que usaram método contraceptivo nas últimas relações sexuais eram mais propensas a serem usuárias dos serviços de planejamento familiar. Aproximadamente 55,0\% das jovens afirmaram que buscariam um serviço especializado para sua faixa etária, ainda que distante de sua residência. Constatou-se a necessidade de investimentos em serviço especializado para jovens, com características próprias que facilitam o acesso precoce, bem como atendam aos anseios das jovens que mesmo tendo vivenciado uma gravidez na adolescência, não utilizam os serviços de planejamento familiar disponíveis. Palavras-chave Serviços de saúde, Planejamento familiar, Gravidez na adolescência 


\section{Introdução}

O uso dos serviços de saúde depende de vários fatores, abrangendo aspectos individuais, contextuais e de interação entre o individuo que procura assistência e o profissional de saúde que a disponibiliza, sendo a qualidade desta assistência fator de influência para a utilização dos serviços ${ }^{1,2}$.

Com a iniciação sexual ocorrendo cada vez mais precocemente a maioria dos adolescentes é sexualmente ativo ${ }^{3,4}$, e demanda por cuidados preventivos com relação à saúde reprodutiva, principalmente devido à necessidade de se reduzir consequências negativas da prática sexual insegura. Entretanto, a maioria dos serviços de saúde não está adequadamente preparada para receber e resolver as necessidades dos jovens ${ }^{5}$.

Ao considerar que as escolhas reprodutivas dos adolescentes têm relevante impacto sobre sua saúde, escolaridade, perspectivas de emprego e transição global para a vida adulta, a prestação e a utilização de serviços de saúde reprodutiva tornam-se imprescindíveis para melhores perspectivas de vida e de participação produtiva na sociedade $^{6}$.

O planejamento familiar permite aos indivíduos espaçarem e limitarem as gestações de acordo com seu desejo, com impacto direto em sua saúde e bem-estar, bem como sobre o resultado de cada gestação, uma vez que permite seu espaçamento adequado, e pode atrasar a gravidez em mulheres jovens, reduzindo os riscos de problemas de saúde e de mortalidade materna e infantil. Além disso, ao reduzir as taxas de gravidez indesejada, o planejamento familiar reduz a necessidade de abortos inseguros, que responde por $13 \%$ da mortalidade materna global ${ }^{7}$. Repercute também no aspecto social, haja vista a maternidade na juventude estar relacionada com educação precária, baixa inserção no mercado de trabalho, baixa autoestima e falta de perspectiva de vida, fatores que contribuem para a perpetuação do ciclo de pobreza ${ }^{8,9}$.

As ações de planejamento familiar brasileiras, no âmbito do Sistema único de Saúde (SUS), são desenvolvidas principalmente pela Estratégia Saúde da Família (ESF), cujas equipes multiprofissionais trabalham com população adstrita visando formação de vínculo entre o serviço e a comunidade. Cabe a estas equipes, além da assistência em planejamento familiar, a integração com outros serviços de atenção à saúde reprodutiva, de pós-parto e aborto, prevenção do câncer do colo do útero e de controle das doenças sexualmente transmissíveis (DST), a fim de pro- mover assistência global à usuária em qualquer contato com o serviço de saúde ${ }^{10,11}$.

Na prática, o planejamento familiar preconizado pelo Ministério da Saúde não é compatível com as ações realizadas pela ESF, uma vez que, apesar de ser considerado prioritário, o planejamento familiar ocupa plano secundário nos serviços de saúde, onde maior ênfase é dada ao ciclo grávido-puerperal ${ }^{11,12}$. Até mesmo o encaminhamento ao atendimento de planejamento familiar é feito principalmente para mulheres que estão no pré-natal ou pós-parto. Não se observa o mesmo empenho para atender as necessidades de mulheres em idade reprodutiva que ainda não possuem antecedente gestacional ou que sejam sexualmente inativas ou ainda, aquelas que tenham dificuldade para engravidar. Mostrando, portanto, que na organização dos serviços não é prioridade oferecer às usuárias a possibilidade de trilhar sua trajetória sexual sem risco de gravidez indesejada ou mesmo ter os filhos que deseje ${ }^{13}$.

Para os adolescentes o problema pode ser ainda maior, pois os serviços de saúde não são organizados para o atendimento em planejamento familiar para este grupo e a procura espontânea é pequena, ocorrendo geralmente quando já estão grávidas e desejam iniciar o pré-natal ${ }^{12,13}$.

No Brasil, no ano de 2009, aproximadamente $1 / 5$ dos nascidos vivos, eram filhos de mães adolescentes. No Piauí, no mesmo ano, esse índice era de quase $23 \%{ }^{14}$. Estudo realizado em Teresina, entre adolescentes com antecedentes obstétricos, mostrou que a maioria das gestações ocorridas não foi planejada ${ }^{15}$.

Países em desenvolvimento são especialmente afetados pela elevada fecundidade adolescente, sendo significativa a diferença quando comparados a países desenvolvidos, o que torna a fecundidade nessa faixa etária um preditor do nível de desenvolvimento do país9. O princípio da equidade afirma que indivíduos diferentes entre si, merecem tratamento diferenciado que elimine ou reduza a desigualdade. Nesse sentido, estudos sobre a utilização de serviços de saúde podem fornecer subsídios para a organização da assistência, visto que possibilitam o estabelecimento de níveis de cobertura e identificação de grupos excluídos ${ }^{16,17}$.

Assim, considerando que os serviços de planejamento familiar têm papel decisivo na abordagem e intervenção da gravidez na adolescência como problema social e de saúde pública, o presente estudo tem como objetivo analisar o uso dos serviços de planejamento familiar por jovens com experiência de gravidez, com o intuito for- 
necer subsídios para o desenvolvimento de estratégias para a melhor assistência às demandas desse grupo populacional.

\section{Metodologia}

Este estudo é um recorte de projeto maior intitulado Gravidez na adolescência: fatores preditores da reincidência, desenvolvido pelo Núcleo de Estudos e Pesquisas sobre Mulher e Relações de Gênero (NEPEM) do Departamento de Enfermagem da Universidade Federal do Piauí (UFPI) e financiado pelo Conselho Nacional de Desenvolvimento Científico e Tecnológico $(\mathrm{CNPq})$.

Trata-se de estudo transversal, realizado com jovens residentes em área urbana de Teresina (PI), cujos dados foram coletados em 2008. Os sujeitos da pesquisa são jovens que finalizaram uma gravidez no primeiro quadrimestre de 2006, quando tinham entre 15 e 19 anos de idade, em seis maternidades do município, sendo cinco públicas e uma privada.

Haja vista que o estudo original pretendia investigar a reincidência de gravidez, optou-se por eleger os casos de finalização de gravidez ocorridos dois anos antes da coleta de dados, com a finalidade de analisar a possível ocorrência de nova gestação nesse prazo. Este período foi escolhido em virtude de ser o intervalo interpartal preconizado pela OMS, como forma de reduzir os riscos de problemas de saúde para mãe e concepto ${ }^{18}$.

Devido ao Sistema de Informação do Programa de Humanização no Pré-Natal e Nascimento (SISPRENATAL) possuir cobertura insatisfatória no estado do Piauí e que o estudo piloto mostrou a inviabilidade da localização dos casos se o acesso inicial se desse pela atenção básica, em decorrência da deficiente organização de suas fontes de informação, optou-se pelos dados gerados nas maternidades. Apesar das potenciais limitações nos registros hospitalares, avaliou-se que essas seriam menores que as detectadas nos serviços de atenção básica.

Em 2006, 19,5\% de todos os partos de mães com residência em Teresina foram de adolescentes, totalizando 2.852 crianças vivas de mães nessa faixa etária ${ }^{19}$. Desse total, a proporção calculada para os quatro primeiros meses do referido ano foi de aproximadamente 950 jovens. Entretanto, como todas as formas de resolução da gravidez seriam incluídas e que nem todas têm dimensão conhecida como os casos de abortamento, o cálculo da amostra probabilística seria inviável. Mas previu-se que o número aproxi- mado de jovens que finalizariam uma gravidez no período determinado para o estudo seria em torno de 1000, verificando-se assim, a viabilidade do estudo aos recursos disponíveis. Optouse, dessa forma, pela amostragem acidental, que independe de desenho amostral, sendo os dados colhidos de acordo com a conveniência na localização dos mesmos.

Em face da metodologia empregada, ser a busca ativa dos casos nos bancos de dados das maternidades dois anos após o seu registro, era esperado que muitos casos não fossem localizados. Também foi considerada limitação da amostragem, a falta de acesso aos dados de curetagem ocorridos no estabelecimento de saúde privado, devido o sigilo que os envolvem, uma vez que podem ser resultado não somente de abortos espontâneos, mas também de provocados. Entretanto, com a análise destas limitações, conclui-se que elas não inviabilizariam o estudo.

Dessa forma, das 632 jovens localizadas nos registros das maternidades, 168 foram considerados casos perdidos, os motivos foram: um óbito materno, três recusas, e 164 casos devido a não localização, totalizando 464 entrevistas realizadas.

O instrumento de coleta de dados foi um formulário estruturado e pré-testado. Os dados foram coletados de maio a dezembro de 2008. As jovens foram identificadas pelos registros das maternidades incluídas no estudo, com posterior contato telefônico para convidá-las a participarem da pesquisa. Quando não era possível o contato telefônico devido à ausência deste dado nos registros, mas o endereço estava disponível, duas entrevistadoras dirigiam-se ao local da residência destas jovens para convidá-las a participarem. Consideraram-se casos perdidos apenas os óbitos maternos, bem como as jovens não encontradas depois de esgotadas todas as estratégias e tentativas de localização.

Os dados foram digitados, inicialmente, no software Epi Info. Versão 6.04d (Centers for Disease Control and Prevention, Atlanta, Estados Unidos), em dois bancos de dados para que fossem checados eventuais erros de digitação e feitas às devidas correções. Para a análise dos dados utilizou-se o software SPSS v.17.0 para Windows.

$\mathrm{Na}$ análise bivariada dos dados, utilizou-se o teste do qui-quadrado de Pearson. Para verificar os fatores associados ao uso dos serviços de planejamento familiar por jovens com antecedente gestacional foi feita análise multivariada desenvolvida por meio da regressão logística múltipla-RLM ajustada para possíveis variáveis de 
confusão usando o método enter e analisando o ajuste do modelo pelo teste de Hosmerand and Lemeshow. Foi obtida então a odds ratio (OR) como medida de efeito, com seu respectivo intervalo de confiança de 95\% (IC95\%).

O critério para inclusão de variáveis no modelo logístico foi a associação com as características socioeconômicas, aspectos reprodutivos, prática contraceptiva e fonte de informação sobre sexualidade em nível de $p<0,20$ na análise bivariada. O critério de permanência das variáveis no modelo foi associação em nível de $\mathrm{p}<0,05$.

O teste de multicolinearidade necessário para a RLM foi feito pelo VIF (Variance Inflation Factor). Adotou-se como ponto de corte para o diagnóstico de multicolinearidade um VIF acima de $4^{20}$. O teste não detectou multicolinearidade entre as variáveis independentes estudadas.

A pesquisa cumpriu os preceitos éticos e legais das pesquisas que envolvem seres humanos, segundo determinação da resolução 196/96 ${ }^{21}$ do Conselho Nacional de Saúde. Foi solicitada a assinatura do Termo de Consentimento Livre e Esclarecido (TCLE) para todas as participantes desta pesquisa. Para jovens menores de 18 anos, o TCLE foi assinado por seu responsável legal.
Anteriormente ao início da coleta de dados, foi requerida permissão de acesso aos dados das pacientes aos gestores da instituição estadual, à Fundação Municipal de Saúde, gestora do Sistema Único de Saúde em Teresina e à direção do estabelecimento privado. O projeto maior, do qual faz parte este estudo, foi aprovado pelo Comitê de Ética em Pesquisa da Universidade Federal do Piauí.

\section{Resultados}

Aproximadamente 70,0\% das entrevistadas estavam na faixa etária dos 20 aos 22 anos. A maioria $(86,9 \%)$ referiu ter companheiro, e menos de $1 \backslash 3$ afirmou estar estudando. Quase metade da amostra tinha baixa escolaridade, com até o ensino fundamental (menos de 8 anos de estudo). Apenas cerca de $1 \backslash 4$ da amostra referiu ter trabalho remunerado, sendo prevalente a baixa renda familiar, uma vez que $50,0 \%$ das participantes vivia com renda de até um salário mínimo. À análise bivariada escolaridade e renda familiar apresentaram associação significativa com o uso do serviço de planejamento familiar (Tabela 1).

Tabela 1. Uso dos serviços de planejamento familiar por jovens com antecedentes gestacionais segundo aspectos sociodemográficos. Teresina (PI). 2008.

\begin{tabular}{|c|c|c|c|c|c|c|c|c|c|}
\hline \multirow{3}{*}{ Variáveis } & & & \multicolumn{6}{|c|}{ Usuária do serviço de planejamento familiar } & \multirow{3}{*}{$\mathbf{p}^{* *}$} \\
\hline & \multicolumn{2}{|c|}{ Distribuiçãao } & \multicolumn{2}{|c|}{ Sim } & \multicolumn{2}{|c|}{ Não } & \multicolumn{2}{|c|}{ Total } & \\
\hline & $\mathbf{n}$ & $\%$ & $\mathbf{n}$ & $\%$ & $\mathbf{n}$ & $\%$ & $\mathbf{n}$ & $\%$ & \\
\hline Faixa etária (anos completos) & & & & & & & & & 0,080 \\
\hline $17-19$ & 140 & 30,2 & 90 & 64,3 & 50 & 35,7 & 140 & 100,0 & \\
\hline $20-22$ & 324 & 69,8 & 180 & 55,6 & 144 & 44,4 & 324 & 100,0 & \\
\hline Tem companheiro & & & & & & & & & 0,487 \\
\hline Sim & 403 & 86,9 & 237 & 58,8 & 166 & 41,2 & 403 & 100,0 & \\
\hline Não & 61 & 13,1 & 33 & 54,1 & 28 & 45,9 & 61 & 100,0 & \\
\hline Estuda & & & & & & & & & 0,675 \\
\hline Sim & 141 & 30,4 & 80 & 56,7 & 61 & 43,3 & 141 & 100,0 & \\
\hline Não & 323 & 69,6 & 190 & 58,8 & 133 & 41,2 & 323 & 100,0 & \\
\hline Escolaridade (anos de estudo) & & & & & & & & & 0,026 \\
\hline$\leq 8$ & 222 & 47,8 & 141 & 63,5 & 81 & 36,5 & 222 & 100,0 & \\
\hline$>8$ & 242 & 52,2 & 129 & 53,3 & 113 & 46,7 & 242 & 100,0 & \\
\hline Trabalha & & & & & & & & & 0,520 \\
\hline Sim & 129 & 27,8 & 72 & 55,8 & 57 & 44,2 & 129 & 100,0 & \\
\hline Não & 335 & 72,2 & 198 & 59,1 & 137 & 40,9 & 335 & 100,0 & \\
\hline Renda familiar & & & & & & & & & $<0,001$ \\
\hline Até $1 \mathrm{SM}^{*}$ & 232 & 50,0 & 147 & 63,4 & 85 & 36,6 & 232 & 100,0 & \\
\hline De 1 a 3 SM & 183 & 39,4 & 111 & 60,7 & 72 & 39,3 & 183 & 100,0 & \\
\hline Mais de $3 \mathrm{SM}$ & 49 & 10,6 & 12 & 24,5 & 37 & 75,5 & 49 & 100,0 & \\
\hline
\end{tabular}

“Salário mínimo (na época da coleta de dados correspondendo a 412,00 R\$). ${ }^{* *}$ Teste qui-quadrado de Pearson. 
profissionais de saúde $(37,5 \%)$, no entanto, $7,7 \%$ das jovens relataram não possuir nenhuma fonte. As variáveis relacionadas aos aspectos reprodutivos, prática contraceptiva e fonte de informação mostram-se significativamente associadas ao uso dos serviços de planejamento familiar (Tabela 2).

Aproximadamente 55,0\% das jovens afirmaram que buscariam um serviço especializado para sua faixa etária, ainda que o mesmo fosse distante de sua residência, sendo as principais razões para essa busca: acreditarem que seriam melhor informadas $(22,0 \%)$, e pela homogeneidade da clientela (17,2\%). Dentre as que não buscariam o serviço especializado os principais motivos são: a possível distância $(26,3 \%)$ e a satisfação com a assistência recebida no posto de saúde que frequentam (13,2\%). O motivo para buscar serviço apresentou significância estatística com o

uso dos serviços de planejamento familiar na análise bivariada (Tabela 3).

Foram incluídas no modelo de regressão logística as variáveis que apresentaram $\mathrm{p}<0,20$ na análise bivariada.

Após ajuste, observou-se que as jovens com renda familiar maior que três salários mínimos apresentaram-se $20 \%$ mais propensas a não serem usuárias dos serviços de planejamento familiar, quando comparadas às que possuíam até um salário mínimo. O número de gestação também se mostrou associado, as jovens que referiram mais de uma gravidez tinham 1,6 vezes mais chances de usarem o serviço. Quando comparadas às jovens que não possuem nenhuma fonte de informação, àquelas que tinham profissionais de saúde ou família como fonte de informação tinha cerca de quatro vezes e meia mais chances de usarem o serviço de saúde, enquanto as que tinham o parceiro como fonte de informação tinham cerca de três vezes mais chance. No que se refere ao uso de métodos contraceptivos, as jovens que referiram estar utilizando tinham três vezes maior propensão a serem usuárias dos serviços de planejamento familiar (Tabela 4).

Tabela 2. Uso do serviço de planejamento familiar por jovens com antecedentes gestacionais segundo aspectos reprodutivos, prática contraceptiva e fonte de informação. Teresina (PI). 2008.

\begin{tabular}{|c|c|c|c|c|c|c|c|c|c|}
\hline \multirow{3}{*}{ Variáveis } & & & \multicolumn{6}{|c|}{ Usuária do serviço de planejamento familiar } & \multirow{3}{*}{$\mathbf{p}^{*}$} \\
\hline & \multicolumn{2}{|c|}{ Distribuição } & \multicolumn{2}{|c|}{ Sim } & \multicolumn{2}{|c|}{ Não } & \multicolumn{2}{|c|}{ Total } & \\
\hline & n & $\%$ & $\mathbf{n}$ & $\%$ & $\mathbf{n}$ & $\%$ & n & $\%$ & \\
\hline Número de gestações & & & & & & & & & 0,017 \\
\hline 1 & 228 & 49,1 & 120 & 52,6 & 108 & 47,4 & 228 & 100,0 & \\
\hline Mais de 1 & 236 & 50,9 & 150 & 63,6 & 86 & 36,4 & 236 & 100,0 & \\
\hline Número de filhos vivos & & & & & & & & & 0,015 \\
\hline Até 1 & 313 & 67,5 & 170 & 54,3 & 143 & 45,7 & 313 & 100,0 & \\
\hline Mais de 1 & 151 & 32,5 & 100 & 66,2 & 51 & 33,8 & 151 & 100,0 & \\
\hline $\begin{array}{l}\text { Uso de método contraceptivo } \\
\text { nas ultimas relações sexuais }\end{array}$ & & & & & & & & & $<0,001$ \\
\hline Sim & 359 & 77,4 & 225 & 62,7 & 134 & 37,3 & 359 & 100,0 & \\
\hline Não & 105 & 22,6 & 45 & 42,9 & 60 & 57,1 & 105 & 100,0 & \\
\hline $\begin{array}{l}\text { Principal fonte de } \\
\text { informação sobre sexualidade }\end{array}$ & & & & & & & & & 0,004 \\
\hline Profissionais de saúde & 174 & 37,5 & 117 & 67,2 & 57 & 32,8 & 174 & 100,0 & \\
\hline Família & 66 & 14,2 & 43 & 65,5 & 23 & 34,8 & 66 & 100,0 & \\
\hline Amigos & 65 & 14,0 & 34 & 52,3 & 31 & 47,7 & 65 & 100,0 & \\
\hline Parceiro & 49 & 10,6 & 29 & 59,2 & 20 & 40,8 & 49 & 100,0 & \\
\hline Escola & 11 & 2,4 & 5 & 45,5 & 6 & 54,5 & 11 & 100,0 & \\
\hline Outras & 63 & 13,6 & 28 & 44,4 & 35 & 55,6 & 63 & 100,0 & \\
\hline Nenhuma & 36 & 7,7 & 14 & 38,9 & 22 & 61,1 & 36 & 100,0 & \\
\hline
\end{tabular}

* Teste qui-quadrado de Pearson. 
Tabela 3. Uso do serviço de planejamento familiar por jovens com antecedentes gestacionais segundo busca por serviço especializado. Teresina (PI). 2008.

\begin{tabular}{|c|c|c|c|c|c|c|c|c|c|}
\hline \multirow{3}{*}{ Variáveis } & & & \multicolumn{6}{|c|}{ Usuária do serviço de planejamento familiar } & \multirow{3}{*}{$\mathbf{p}^{*}$} \\
\hline & \multicolumn{2}{|c|}{ Distribuição } & \multicolumn{2}{|c|}{ Sim } & \multicolumn{2}{|c|}{ Não } & \multicolumn{2}{|c|}{ Total } & \\
\hline & $\mathbf{n}$ & $\%$ & $\mathbf{n}$ & $\%$ & $\mathbf{n}$ & $\%$ & $\mathbf{n}$ & $\%$ & \\
\hline $\begin{array}{l}\text { Buscaria serviço especializado } \\
\text { para adolescentes }\end{array}$ & & & & & & & & & 0,198 \\
\hline Sim & 254 & 54,8 & 141 & 55,5 & 113 & 44,5 & 254 & 100,0 & \\
\hline Não & 210 & 45,2 & 129 & 61,4 & 81 & 38,6 & 210 & 100,0 & \\
\hline $\begin{array}{l}\text { Por que buscar ou não este } \\
\text { serviço }\end{array}$ & & & & & & & & & $<0,001$ \\
\hline \multicolumn{10}{|l|}{ Buscaria } \\
\hline Seria melhor informada & 102 & 22,0 & 50 & 49,0 & 52 & 51,0 & 102 & 100,0 & \\
\hline $\begin{array}{l}\text { Homogeneidade da } \\
\text { clientela }\end{array}$ & 80 & 17,2 & 52 & 65,0 & 28 & 35,0 & 80 & 100,0 & \\
\hline $\begin{array}{l}\text { Qualidade deficiente do } \\
\text { atendimento no posto }\end{array}$ & 55 & 11,9 & 32 & 58,2 & 23 & 41,8 & 55 & 100,0 & \\
\hline $\begin{array}{l}\text { Não haveria vizinhos para } \\
\text { comentar }\end{array}$ & 14 & 3,0 & 6 & 42,9 & 8 & 57,1 & 14 & 100,0 & \\
\hline \multicolumn{10}{|l|}{ Não buscaria } \\
\hline Distância & 122 & 26,3 & 77 & 63,1 & 45 & 36,9 & 122 & 100,0 & \\
\hline O posto é próximo e bom & 61 & 13,2 & 47 & 77,0 & 14 & 23,0 & 61 & 100,0 & \\
\hline Não é usuária do SUS & 15 & 3,2 & 2 & 13,3 & 13 & 86,7 & 15 & 100,0 & \\
\hline Indiferente & 9 & 1,9 & 2 & 22,2 & 7 & 77,8 & 9 & 100,0 & \\
\hline Outros & 6 & 1,3 & 2 & 33,3 & 4 & 66,7 & 6 & 100,0 & \\
\hline
\end{tabular}

* Teste qui-quadrado de Pearson.

\section{Discussão}

As características sociodemográficas dos indivíduos apresentam-se como um dos determinantes para a utilização dos serviços de saúde públi$\cos ^{1}$. No presente estudo a renda apresentou relevante associação com o uso dos serviços públicos de planejamento familiar, os quais são utilizados principalmente por pessoas de menor renda e geralmente sem acesso a planos de saúde e serviços médicos privados.

Tem sido mostrado que a utilização de planos de saúde está diretamente relacionada à maior renda familiar ${ }^{22}$, bem como os serviços de saúde públicos são majoritariamente utilizados por indivíduos de menor renda. Estudo realizado em Porto Alegre (RS) verificou que a probabilidade de utilização da unidade de saúde da família local foi cinco vezes maior entre pessoas de menor nível socioeconômico $(16,4 \%)$, em relação às de maior nível socioeconômico (3,0\%), mostrando uma tendência linear significativa ${ }^{17}$.

É notória a diferença na fecundidade nas diferentes classes sociais, com os indivíduos de menor renda apresentando maior necessidade insa- tisfeita de anticoncepção. Nesse sentido, promover o equilíbrio entre renda e fecundidade por meio do planejamento reprodutivo, torna-se imprescindível para a diminuição da pobreza e da desigualdade social, com melhorias na saúde materna e infantil, e na condição social da população ${ }^{23}$.

No Peru, a ampliação dos serviços e da disponibilidade de anticoncepcionais colaborou com o incremento no uso de contraceptivos entre $1996 \mathrm{e}$ 2000 , de $41 \%$ para $44 \%$, sendo o aumento na prevalência de uso de métodos modernos entre as mulheres de menor renda de $18 \%$ para $25 \%{ }^{6}$. No Brasil também houve aumento nas taxas de uso de métodos contraceptivos entre 1996 e 2006, principalmente devido à expansão nos estratos de menor renda, com queda de $35,1 \%$ para $26,3 \%$ na taxa de não uso de métodos contraceptivos nas mulheres em união conjugal da classe $\mathrm{E}^{3}$.

Os resultados constatados no presente estudo estão de acordo com o pressuposto de que a mudança do modelo assistencial e a implantação da Estratégia de Saúde da Família tendem a minimizar as desigualdades sociais e em saúde, mediante a melhora das condições de saúde da população de menor renda ${ }^{17}$. Percebe-se, portan- 
Tabela 4. Modelo de regressão logística binomial para o uso dos serviços de planejamento familiar por jovens com antecedentes gestacionais. Teresina (PI), 2008.

\begin{tabular}{|c|c|c|}
\hline Variáveis & ORa (IC95\%) & $\mathbf{p}^{*}$ \\
\hline \multicolumn{3}{|l|}{ Faixa etária } \\
\hline 17-19 anos & 1 & \\
\hline 20-22 anos & $0,7(0,4-1,1)$ & 0,134 \\
\hline \multicolumn{3}{|l|}{ Escolaridade } \\
\hline$\leq 8$ anos & 1 & \\
\hline$>8$ anos & $0,8(0,5-1,2)$ & 0,292 \\
\hline \multicolumn{3}{|l|}{ Renda familiar } \\
\hline Até $1 \mathrm{SM}$ & 1 & \\
\hline 1-3 SM & $0,9(0,6-1,5)$ & 0,778 \\
\hline$>3 \mathrm{SM}$ & $0,2(0,1-0,4)$ & $<\mathbf{0 , 0 0 1}$ \\
\hline \multicolumn{3}{|l|}{ Número de gestações } \\
\hline Uma & 1 & \\
\hline Mais de uma & $1,6(1,1-2,5)$ & 0,029 \\
\hline \multicolumn{3}{|l|}{ Número de filhos } \\
\hline Até um & 1 & \\
\hline Mais de um & $1,1(0,6-2,0)$ & 0,765 \\
\hline \multicolumn{3}{|c|}{ Uso de métodos contraceptivos nas ultimas relações sexuais } \\
\hline Não & 1 & \\
\hline Sim & $3,0(1,8-4,8)$ & $<0,001$ \\
\hline \multicolumn{3}{|l|}{ Fonte de informação sobre sexualidade } \\
\hline Nenhuma & 1 & \\
\hline Amigos & $2,3(1,0-5,4)$ & 0,060 \\
\hline Família & $4,4(1,8-10,7)$ & 0,001 \\
\hline Parceiro & $3,1(1,2-7,9)$ & 0,015 \\
\hline Escola & $2,3(0,5-9,7)$ & 0,272 \\
\hline Profissionais de saúde & $4,5(2,1-9,8)$ & $<\mathbf{0 , 0 0 1}$ \\
\hline Outros & $1,8(0,7-4,3)$ & 0,200 \\
\hline \multicolumn{3}{|c|}{ Buscaria serviço especializado para adolescente } \\
\hline Não & 1 & \\
\hline Sim & $0,8(0,5-1,2)$ & 0,341 \\
\hline
\end{tabular}

Ajuste para todas as variáveis. "Teste de Wald significativo. IC95\% = intervalo de confiança de 95\%; ORa = razão de chances ajustado (oddsratio). Teste de Hosmer e Lemeshow, $\mathrm{p}=0,707$

to, a necessidade e a importância de se ampliar a disponibilidade de serviços de planejamento familiar para a população de menor renda, a qual possui maior dificuldade de controle de fecundidade, como forma de intervir para o melhoramento da qualidade e das perspectivas de vida deste grupo.

Outro possível motivo para a menor utilização dos serviços de planejamento familiar pelas jovens de maior renda é o fato de que grande parte destas estuda e/ou trabalha (dados não mostrados), o que pode resultar em menor tempo e disposição para buscar os serviços de saúde. Como constatado em pesquisa realizada em São Paulo (SP), que verificou que a procura por serviços de saúde, por qualquer motivo, é igual ou menor entre mulheres trabalhadoras em comparação com donas de casa ${ }^{24}$.
No momento da entrevista, pouco mais da metade das jovens referiu ter tido mais de uma gestação. Esta característica mostrou-se associada ao uso dos serviços de planejamento familiar. Maior uso dos serviços de saúde reprodutiva por mulheres com maior número de gestações também foi observada no México, indicando que, potencialmente, essas mulheres procuram o planejamento familiar para cessar sua fertilidade, e não para limitar o número de filhos ao desejado, ou promover o espaçamento adequado entre as gestações. Revelando que, para as jovens, ter experiência de repetição de gravidez garante maior acesso ao planejamento reprodutivo, facilitando a disposição para o controle da natalidade ${ }^{13,25}$.

Portanto, ser multigesta foi a característica das participantes do presente estudo que as impulsionaram a buscar os meios de ter a decisão 
sobre o número de filhos a partir daquele momento de suas vidas. A ocorrência de uma gravidez não foi suficiente para garantir a utilização do serviço de planejamento familiar por estas jovens, o qual só ocorreu significativamente após vivenciarem mais de uma gestação. Desta forma, supõe-se, que a ocorrência de uma gravidez pode ser até considerado algo positivo para e pela jovem, mas quando ocorre sua repetição as jovens recorrem aos serviços de saúde em busca de atender sua necessidade de ajuda para o controle reprodutivo. Entretanto, visto que o motivo das jovens buscarem o serviço de saúde para controle reprodutivo não foi questionado no presente estudo, sugere-se que estudos longitudinais identifiquem se há de fato variações nos motivos de busca ou não a esses serviços durante a vida reprodutiva de jovens.

$\mathrm{O}$ uso de algum método contraceptivo nas ultimas relações sexuais foi relatado pela maioria das jovens. Ter usado algum método contraceptivo nas últimas relações sexuais mostrou-se associado ao uso do serviço de planejamento familiar. Associação também constatada entre mulheres mexicanas ${ }^{25}$. A prática da contracepção nas últimas relações aponta para o potencial desempenho a contento do serviço de saúde em seu papel de favorecer o controle da fertilidade entre as jovens que o buscam.

No Brasil, a necessidade insatisfeita de planejamento familiar caiu de $12,8 \%$ em 1986, para $6,0 \%$ em 2006, enquanto o uso de métodos contraceptivos modernos cresceu de $56,7 \%$ para $77,1 \%$ no mesmo período ${ }^{26}$. Mostrando novamente que o aumento da disponibilidade de assistência em planejamento familiar aumenta o uso de métodos contraceptivos, e reforçando a importância da ampliação desses serviços como forma de intervir na problemática das gestações indesejadas, principalmente entre adolescentes.

A análise multivariada também apontou que, quando comparadas às jovens que não possuem nenhuma fonte de informação, aquelas que citaram profissionais de saúde, família ou parceiro como fonte de informação tinham mais propensão a serem usuárias do serviço de planejamento familiar. Este resultado aponta a relevância de uma rede de apoio social para a busca e a utilização consistente de serviços de saúde reprodutiva e consequentemente da adoção de práticas contraceptivas adequadas.

Várias pesquisas realizadas com adolescentes e jovens em geral mencionam como principal fonte de informação amigos e parentes próximos, e apontam os profissionais de saúde como fontes pouco utilizadas, principalmente pelo receio de julgamentos e da falta de confidencialida$\mathrm{de}^{27,28}$. Todavia, estudo realizado em Teresina, com adolescentes com antecedente gestacional, confirma os profissionais de saúde como importante fonte de informação ${ }^{29}$. Nota-se que após a ocorrência de uma gestação, a jovem fica menos constrangida em buscar auxilio dos profissionais de saúde para questões relacionadas ao sexo $^{30}$, e demonstra que uma vez engajadas em serviço de planejamento familiar a jovem tende a percebê-lo como importante provedor de informações e, consequentemente, práticas reprodutivas saudáveis. Diante deste achado, nota-se a necessidade de mudança no modelo de assistência atualmente prestado, que espera ser procurado, por um modelo que busque os adolescentes precocemente, agindo de forma preventiva.

No que se refere à família, a literatura mostra que nas famílias em que existe espaço de diálogo sobre sexualidade, nota-se um sinal de confiança mútua, pois os pais passam a fazer parte dos recursos de aprendizagem de tais conteúdos, independentemente do tipo de dúvida, e se favorece o uso consistente de contraceptivos pelas adolescentes $^{27}$ A referência da mãe como fonte de informação sobre sexualidade está relacionada com uma diminuição na crença de que fazer sexo não traz resultados negativos, como gravidez indesejada e contágio por alguma DST ${ }^{31}$.

Para os jovens, ter comunicação positiva com os pais proporciona suporte emocional e sentimento de apoio sendo considerado fator protetor $^{32}$. Percebe-se, portanto, a importância do apoio da família nas ações de promoção da saúde sexual e reprodutiva de adolescentes e jovens, por meio do incentivo ao uso dos serviços de planejamento familiar e consequentemente dos métodos contraceptivos.

A literatura mostra que adolescentes sem companheiro têm maior receio de procurar serviços de saúde reprodutiva ${ }^{33}$. No presente estudo, apesar de não ter sido verificada relação entre o uso dos serviços de planejamento familiar e ter ou não companheiro, observou-se que ter o parceiro como fonte de informação sobre sexualidade está associado ao uso desses serviços. Ter comunicação e apoio do parceiro para o uso de contraceptivos está associado à utilização de serviços de planejamento familiar, apontando a importância de ampliar a participação masculina nos programas de saúde reprodutiva ${ }^{25}$.

Deve-se lembrar, portanto, que a gravidez na adolescência não é um acontecimento exclusivamente feminino, sendo imperativo oferecer mais 
atenção ao gênero masculino, como forma de buscar uma resolução para o problema ${ }^{34}$, inclusive mediante a mudança de cultura das ações de planejamento familiar e saúde reprodutiva que são, na maioria das vezes, voltadas unicamente para mulheres ${ }^{35}$.

Quando questionadas se utilizariam serviço de saúde especializado para jovens, mesmo este sendo longe de suas casas, a maior parte das entrevistadas respondeu afirmativamente, sem divergência entre usuárias e não usuárias dos serviços próximos. Contudo o motivo citado pelas participantes para buscar ou não este serviço especializado apresentou diferenças significativas entre os dois grupos.

Entre os motivos para buscar serviço especializado, destacou-se, entre as usuárias, a homogeneidade da clientela no novo serviço. Já entre as não usuárias a preferência pelo serviço especializado deve-se à ausência dos comentários de vizinhos. O motivo predominante para não buscar o serviço especializado entre as usuárias foi estar satisfeita com a assistência que recebem no serviço próximo às suas casas, e entre as não usuárias o principal motivo foi não ser usuária do SUS.

Nota-se por esse resultado, que a existência desse serviço especializado seria de grande valia para a população jovem. Características valorizadas pelos jovens como: homogeneidade da clientela, privacidade na busca por atendimento, além de outras que certamente seriam facilitadoras da utilização desse serviço, como garantia de confidencialidade e autonomia, melhor acolhimento, profissionais treinados para o atendimento a este tipo de clientela, horários flexíveis e multiplicidade de serviços prestados. Desta forma, seriam atendidas as diversas demandas existentes, não só as referentes à saúde sexual e reprodutiva, em um só lugar, e de uso exclusivo dessa clientela, que carece de atenção especial por parte dos gestores e profissionais de saúde.

Para a realidade brasileira, devido às grandes diferenças regionais, vale acrescentar a necessidade de respeito às características socioeconômicas e culturais da co-munidade jovem usuária, além de seu perfil epidemiológico ${ }^{36}$.
A existência de serviços especializados para adolescentes facilita a acessibilidade e a utilização dos serviços de saúde por essa clientela e melhora a qualidade dos cuidados prestados, com implicações na prevenção da gravidez indesejada e todos os riscos associados a ela ${ }^{37}$. Para tal, além das características já citadas, soma-se a necessidade da união entre os setores saúde e educação e os demais ligados à juventude, e de aprimorar a publicidade dos benefícios para dissolver o medo e a ansiedade quanto ao uso dos serviços de saú$\mathrm{de}^{9,33}$. Além disso, devem ser realizadas ações e atividades estratégicas, tanto no interior das unidades de saúde quanto nas comunidades para captação dos jovens ${ }^{36}$.

\section{Considerações finais}

A assistência em planejamento familiar é de primordial importância para a experiência de vida sexual e reprodutiva saudável, especialmente entre adolescentes e jovens que devido aos comportamentos de risco estão mais expostos às consequências negativas de práticas sexuais inseguras, sejam elas no âmbito biológico, psicológico ou social. Assim, faz-se necessário engajá-los precocemente nos serviços de planejamento familiar, antes da ocorrência e recorrência de gravidez, para que possam ter capacidade de prevenir uma indesejada, bem como, controlar sua fecundidade ao número de filhos que desejam. E que estes venham de forma planejada, em contexto socioeconômico favorável para seu nascimento e desenvolvimento.

$\mathrm{Na}$ literatura há poucos trabalhos que abordam o uso dos serviços de saúde por jovens. Nacionalmente esse número é ainda menor, não havendo dados sobre a utilização dos serviços de saúde reprodutiva por jovens. Percebe-se assim a relevância do presente estudo, em mostrar o panorama da situação. Neste sentido, fica clara a necessidade de explorar o tema, de modo que se possa entender e atender as necessidades dos jovens, assim como acompanhar e avaliar a evolução da assistência prestada e a repercussão a curto e a longo prazo na vida dos jovens. 


\section{Colaboradores}

LNB Moura realizou a análise e interpretação dos dados e redação do artigo. KRO Gomes trabalhou na concepção e delineamento da pesquisa e na revisão crítica do artigo.

\section{Referências}

1. Travassos C, Martins M. Uma revisão sobre os conceitos de acesso e utilização de serviços de saúde. Cad Saude Publica 2004; 20(Supl. 2):S190-198.

2. Atuyambe L, Mirembe F, Tumwesigye NM, Annika J, Kirumira EK, Faxelid E. Adolescent and adult first time mothers' health seeking practices during pregnancy and early motherhood in Wakiso district, central Uganda. Reprod Health 2008; 5:13.

3. Brasil. Ministério da Saúde (MS). Centro Brasileiro de Análise e Planejamento. Pesquisa Nacional de Demografia e Saúde da Criança e da Mulher: Dimensões do Processo Reprodutivo e da Saúde da Criança - PNDS 2006. [página na Internet]. [acessado 2011 maio 22]. Disponível em: http://bvsms. saude.gov.br/bvs/pnds

4. Oliveira DC. Iniciação sexual na adolescência e sua repercussão na vida sexual e reprodutiva de jovens [dissertação]. Teresina: Universidade Federal do Piauí; 2011.

5. Ralph LJ, Brindis CD. Access to reproductive healthcare for adolescents: establishing healthy behaviors at a critical juncture in the lifecourse. Curr Opin Obstet Gynecol 2010; 22(5):369-374.

6. Gribble JN, Sharma S, Menotti EP. Family Planning Policies and Their Impacts On the Poor: Peru's Experience. Int Fam Plan Perspect 2007; 33(4):176-181.

7. World Health Organization (WHO). Fact sheet on family planning. Family planning. Ficha NFact sheet $\mathrm{N}^{\circ} 351$. Abril 22011b. [página na Internet]. [acessado 2011 abr 26]. Disponível em: http://www.who.int/ mediacentre/factsheets/fs351/en/index.html

8. Gomes KRO, Speizer IS. Longitudinal study on selfesteem among recently pregnant Brazilian adolescents. Journal of Reproductive and Infant Psychology 2010; 28(4):359-371.

9. Molina RC, Roca CG, Zamorano JS, Araya EG. Family planning and adolescent pregnancy. Best Pract Res Clin Obstet Gynaecol 2010; 24(2):209-222.

10. Brasil. Ministério da Saúde (MS). Área Técnica de Saúde da Mulher. Assistência em Planejamento Familiar: Manual Técnico, Secretaria de Políticas de Saúde. 4a Edição. Brasília: MS; 2002.

11. Moura ERF, Silva RM, Galvão MTG. Dinâmica do atendimento em planejamento familiar no Programa Saúde da Família no Brasil. Cad Saude Publica 2007; 23(4):961-970.

12. Osis MJD, Faúndes A, Makuch MY, Mello MB, Sousa MH, Araújo MJO. Atenção ao planejamento familiar no Brasil hoje: reflexões sobre os resultados de uma pesquisa. Cad Saude Publica 2006; 22(11):24812490.

13. Heilborn ML, Portella AP, Brandão ER, Cabral CS, Grupo CONPRuSUS. Assistência em contracepção e planejamento reprodutivo na perspectiva de usuárias de três unidades do Sistema Único de Saúde no Estado do Rio de Janeiro, Brasil. Cad Saude Publica 2009; 25(Supl. 2):S269-S278. 
14. Brasil. Ministério da Saúde (MS). Informações em saúde. Brasília, 2009. [página na Internet]. [acessado 2012 jan 18]. Disponível em: http://www.datasus. gov.br.

15. Gomes KRO, Speizer IS, Oliveira DD, Moura LNB, Gomes FM. Contraceptive Method Use by Adolescents in Brazilian State Capital. J Pediatr Adolesc Gynecol 2008; 21(4):213-219.

16. Dias-da-Costa JS, Gigante DP, Horta BL, Barros FC, Victora CG. Utilização de serviços de saúde por adultos da coorte de nascimentos de 1982 a 2004-5, Pelotas, RS. Rev Saude Publica 2008; 42(Supl. 2):51-59.

17. Fernandes LCL, Bertoldi AD, Barros AJD. Utilização dos serviços de saúde pela população coberta pela Estratégia de Saúde da Família. Rev Saude Publica 2009; 43(4):595-603.

18. World Health Organization (WHO). Report of WHO technical consultation on birth spacing. Geneva, Switzerland, 2005. [página na Internet]. [acessado 2012 jul 3]. Disponível em: http://www.who.int/ making_pregnancy_safer/documents/birth_spacing. pdf

19. Brasil. Ministério da Saúde (MS). Informações em saúde. Brasília, 2006. [página na Internet]. [acessado 2008 jan 22]. Disponível em: http://www.datasus. gov.br

20. Pallant JF. SPSS survival manual: a step by step guide to data analysis using SPSS. Austrália, 2005. [página na Internet]. [acessado 2012 abr 20]. Disponível em: http://210.212.115.113:81/Amarnath\%20 Bose/Lib/SPSS/SPSS_Survival_Manual_334.pdf

21. Brasil. Ministério da Saúde (MS). Conselho Nacional de Saúde. Resolução no 196 de 10 de outubro de 1996. Diretrizes e Normas Regulamentadoras de Pesquisas Envolvendo Seres Humanos. Diário Oficial da União 1996; 16 out.

22. Travassos C, Oliveira EXG, Viacava F. Desigualdades geográficas e sociais no acesso aos serviços de saúde no Brasil: 1998 e 2003. Cien Saude Colet 2006; 11(4):975-986.

23. Santos JC, Freitas PM. Planejamento familiar na perspectiva do desenvolvimento. Cien Saude Colet 2011; 16(3):1813-1820.

24. Gomes KRO, Tanaka ACA. Morbidade referida e uso dos serviços de saúde por mulheres trabalhadoras, Município de São Paulo. Rev Saude Publica 2003; 37(1):75-82.

25. Estrada F, Hernández-Girón C, Walker D, Campero L, Hernández-Prado B, Maternowska C. Uso de servicios de planificación familiar de la Secretaría de Salud, poder de decisión de la mujer y apoyo de la pareja. Salud pública Méx 2008; 50(6):472-481.

26. United Nations (UN). Department of Economic and Social Affairs, Population Division, 2011. World Contraceptive Use 2010. [página na Internet]. [acessado 2012 dez 2]. Disponível em: http://www.un.org/ esa/population/publications/wcu2010/WCP_2010/ Data.html.
27. Borges ALV, Nichiata LYI, Schor N. Conversando sobre sexo: a rede sociofamiliar como base de promoção da saúde sexual e reprodutiva de adolescentes. Rev Latino-Am Enfermagem 2006; 14(3):422-427.

28. Agampodi SB, Agampodi TC, Piyaseeli UKD. Adolescents perception of reproductive health care services in Sri Lanka. BMC 2008; 8:1-8.

29. Moura LNB, Gomes KRO, Rodrigues, MTP, Oliveira DC. Informação sobre contracepção e sexualidade entre adolescentes que vivenciaram uma gravidez. Acta Paul Enferm 2011; 24(3):320-326.

30. Lemay CA, Cashman SB, Elfenbein DS, Felice ME. Adolescent mother's attitudes toward contraceptive use before and after pregnency. J Pediatr Adolesc Gynecol 2007; 20(4):233-240.

31. Bleakley A, Hennessy M, Fishbein M, Coles Jr HC, Jordan A. How Sources of Sexual Information Relate to Adolescents' Beliefs about Sex. Am J Health Behav 2009; 33(1):37-48.

32. Dias S, Matos MG, Gonçalves A. Percepção dos adolescentes acerca da influência dos pais e pares nos seus comportamentos sexuais. Aná. Psicológica 2007; 25(4):625-634.

33. Nath A, Garg S. Adolescent friendly health services in India: A need of the hour. Indian J Med Sci 2008; 62(11):465-472.

34. Carvalho GM, Merighi MAB, Jesus MCP. Recorrência da parentalidade na adolescência na perspectiva dos sujeitos envolvidos. Texto Contexto Enferm 2009; 18(1):17-24.

35. Costa MCO, Lima IC, Martins Júnior DF, Santos CAST, Araújo FPO, Assis DR. Gravidez na adolescência e co-responsabilidade paterna: trajetória sociodemográfica e atitudes com a gestação e a criança. Cien Saude Colet 2005; 10(3):719-727.

36. Brasil. Ministério da Saúde (MS). Secretaria de Atenção à Saúde. Saúde integral de adolescentes e jovens: orientações para a organização de serviços de saúde. Brasília: MS; 2005.

37. Dickson KE, Ashton J, Smith JM. Does setting adolescent-friendly standards improve the quality of care in clinics? Evidence from South Africa. Int $J$ Qual Health Care 2007; 19(2):80-89.

Artigo apresentado em 08/05/2013

Aprovado em 25/06/2013

Versão final apresentada em 03/07/2013 